\title{
CONTROLE DE CAOS NO MODELO NEURONAL DE HINDMARSH-ROSE COM PARÂMETROS INCERTOS
}

\section{CHAOS CONTROL IN THE HINDMARSH-ROSE NEURON MODEL WITH UNCERTAIN PARAMETERS}

\author{
Raildo Santos de Lima ${ }^{1}$, Fábio Roberto Chavarette ${ }^{2}$ \\ ${ }^{1}$ Universidade Federal de Mato Grosso do Sul - UFMS, Departamento de Matemática, \\ Paranaíba-MS. ${ }^{2}$ Universidade Estadual Paulista - UNESP, Faculdade de Engenharia de \\ Illa Solteira, Departamento de Matemática. \\ E-mail: raildo.lima@ufms.br
}

\begin{abstract}
RESUMO - Na bioengenharia existe uma grande motivação no estudo do modelo neuronal de Hindmarsh-Rose (HR) pelo fato de ser bem representativo ao neurônio biológico podendo, assim, simular vários comportamentos de um neurônio real, dentre eles, o comportamento periódico, aperiódico e caótico. Baseado neste modelo, este artigo propõe a aplicação do projeto de controle linear ótimo ao comportamento incerto e caótico estabelecidos por modificações nos parâmetros do sistema. Para tanto, apresenta-se o sistema matemático do modelo $\mathrm{RH}$ e seu comportamento caótico e posteriormente modificam-se os parâmetros fixos para incertos e investiga-se a dinâmica caótica do sistema. Finalizando, propõe-se a aplicação do controle linear ótimo como método para controlar o comportamento caótico do modelo onde as simulações numéricas são apresentadas para demonstrar a eficácia desta proposta.
\end{abstract}

Palavras-chave: Caos; Parâmetros Incertos; Controle Ótimo.

ABSTRACT - In bioengineering there is a great motivation in studying the Hindmarsh-Rose (HR) neuron model due to the fact that it represents well the biological neuron, making it possible to simulate several behaviors of a real neuron, including periodic, aperiodic and chaotic behaviors, for example. Based on this model, this article proposes applying a linear optimal control design to the uncertain and chaotic behavior established by changes in the parameters of the system. To do so, the mathematical system of the RH model and its chaotic behavior are presented; afterwards, the fixed parameters are replaced by uncertain ones, and the chaotic dynamics of the system is investigated. At last, the linear optimal control is proposed as a method for controlling the chaotic behavior of the model, and numerical simulations are presented to show the efficiency of this proposal.

Keywords: Chaos; Uncertain Parameters; Optimal Control.

\section{INTRODUÇÃO}

Todas as atividades físicas conscientes e inconscientes são controladas pelo sistema nervoso. Ele é formado por bilhões de células nervosas que captam informações vindas do interior e exterior do corpo humano. As células 
nervosas, juntamente com as musculares, têm duas propriedades fisiológicas características, que são a excitabilidade e condutibilidade, ou seja, capacidade de reagir a um dado estímulo e transmitir esse estímulo sob a forma de impulsos eletroquímicos ao longo de suas membranas. A membrana das células nervosas detém propriedades elétricas que são dadas através do arranjo molecular de seus componentes. Essas propriedades estabelecem a sequência de alterações de voltagem produzidas pelo fluxo de corrente através das membranas e sensibilizam a capacidade de células, eletricamente excitáveis, na condução das informações. Por este fato, a membrana do neurônio é capaz de ser comparada a um circuito elétrico que abrange várias unidades repetidas. Cada unidade integra uma determinada quantidade de baterias, um para cada tipo de íon. Cada bateria iônica no análogo elétrico se conecta às fases extra e intracelular por uma resistência variável, que simboliza a permeabilidade da membrana referente à cada íon em questão. Hodgkin e Huxley, em 1952, externaram uma sequência de experimentos no intuito de averiguar o fluxo de corrente elétrica sobre a superfície da membrana da fibra nervosa de uma lula gigante. Com isso, os autores desenvolveram um modelo matemático para o comportamento da membrana podendo ser melhor compreendida a condução e excitação da fibra. (HODGKIN; HUXLEY, 1952).

Tendo como base o modelo de Hodgkin e Huxley, pesquisadores desenvolveram outros modelos aplicáveis à outros tipos de células nervosas como por exemplo, o modelo neuronal de Hindmarsh-Rose (HR), que simula a transmissão de impulsos nervosos no neurônio humano, é um modelo de sistema caótico (DE LIMA CHAVARETTE; ROÉFERO, 2019). Os sistemas caóticos são sistemas dinâmicos caracterizados pela alta sensibilidade às condições iniciais (MONTEIRO, 2002). Esta alta sensibilidade às condições iniciais infunde ao sistema não linear a característica de instabilidade. Esta instabilidade implica em sensibilidade a perturbações gerando, assim, resultados que são imprevisíveis. Esta natureza sensível dos sistemas caóticos é comumente na literatura chamada de efeito borboleta, fenômeno formulado através de equações matemáticas por Edward Lorenz em 1963 (LORENZ, 1963).

$O$ modelo neuronal de $H R$ é, mais especificamente, um modelo de sistema hipercaótico (DE LIMA; CHAVARETTE; ROÉFERO, 2019) cujo comportamento caótico é biologicamente comparado a um indivíduo com epilepsia (PALUS et al., 2001), fato que o torna de grande interesse para este trabalho, que tem como principal objetivo a aplicação de um controlador que elimine seu comportamento caótico e estabilize suas vibrações incertas (CHAVARETTE, 2013).

É de grande interesse na engenharia mecânica controlar sistemas caóticos. Na prática, controlar tais sistemas, significa desenvolver leis de controle de feedback de estado que estabilizem o sistema em torno de pontos de equilíbrio instáveis (JUN et al., 2008). Neste ensejo, muitas técnicas de controle podem ser usadas para controlar um determinado sistema e cada técnica dispõe de peculiaridades e resultados distintos. O método SDRE (State Dependent Riccati Equation) será utilizado para diminuir a oscilação do sistema em torno de uma órbita desejada. Este método foi proposto pela primeira vez por Pearson (PEARSON, 1962) e mais tarde expandido por Wernli e Cook (WERNLI; COOK, 1975). A aplicação da Equação de Riccati Dependente do Estado (SDRE) para controle do sistema não-linear tem sido de crescente aplicação na problemática de controle de sistemas caóticos (MRACEK; CLONTIER; D'SOUZA, 1996; SHAWKY et al., 2007; CLOUTIER; D'SOUZA; MRACEK, 1996).

Este trabalho está organizado da seguinte maneira: na seção 3 , apresenta-se o modelo de Hindmarsh-Rose (HINDMARSH; ROSE, 1984), na seção 4 as ferramentas e técnica utilizadas, na seção 5 o projeto de controle de incertezas, na seção 6 os principais resultados obtidos e, por fim, na seção 7 as considerações finais.

\section{OBJETIVO DA PESQUISA}

O principal objetivo deste trabalho de pesquisa é propor a aplicação do projeto de controle linear ótimo ao comportamento incerto e caótico estabelecidos por modificações nos parâmetros do sistema. Especificamente, apresenta-se o sistema matemático do modelo $\mathrm{RH}$ e seu comportamento caótico, posteriormente modificam-se os parâmetros fixos para incertos investigando a dinâmica caótica do sistema após estas modificações. Por fim, propõe-se a aplicação do controle linear ótimo como método para controlar o comportamento caótico do modelo onde as simulações numéricas declaram a eficácia desta proposta. 


\section{MODELO DE HINDMARSH-ROSE}

Quando um neurônio recebe algum tipo de estímulo, químico, mecânico ou elétrico, pode ocorrer uma alteração da permeabilidade da membrana, permitindo grande entrada de sódio e pequena saída de potássio na célula nervosa. Esse fato sucede de uma inversão das cargas ao redor da membrana fazendo com que fique despolarizada, fato que leva a um potencial de ação. De acordo com a quantidade de íons no meio intracelular essa diferença de potencial varia tendo um valor estacionário de $\quad-70 \mathrm{mV}$. A caracterização do impulso nervoso se dá pela propagação dessa despolarização ao longo do neurônio e assim, posteriormente a passagem do impulso, a membrana sofre uma repolarização recuperando seu estado normal de repouso cessando a transmissão do impulso (BEAR; CONNORS; PARADISO, 2002).

Estão ilustradas na Figura 1 as principais partes do neurônio. Os Dendritos são ramificações que permitem que o neurônio receba estímulos oriundos de outras células. 0 Axônio é o prolongamento do corpo celular por onde se propaga um impulso nervoso para que o mesmo interaja com outros neurônios ou fibras musculares. A Bainha de Mielina tem como principal função proteger o Axônio e também acelerar a velocidade da condução do impulso nervoso (BEAR; CONNORS; PARADISO, 2002).

A formação de burstings neuronais tem sido amplamente estudada no cenário do modelo neuronal de Hindmarsh-Rose (HR), o qual determina uma variável de estado adimensional

Figura 1. Modelo Biológico

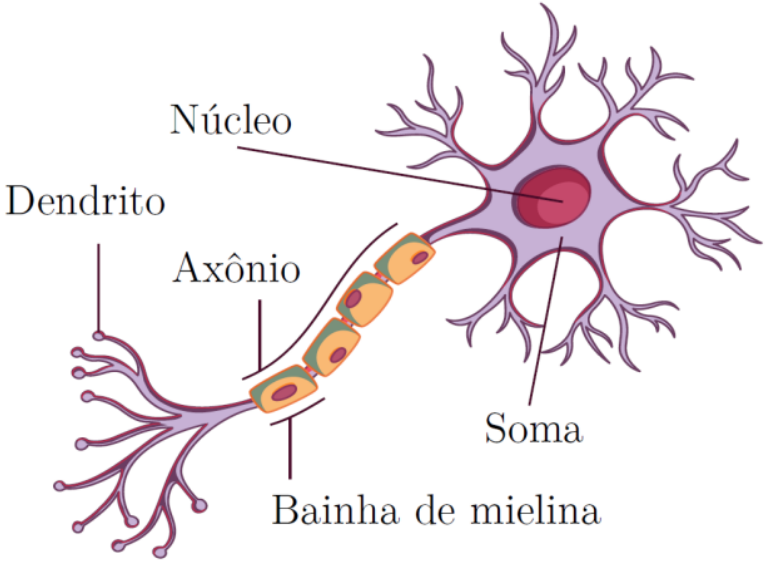

(a) para o potencial de membrana $x(\mathrm{t})$ e outras duas (também adimensionais), $y(t)$ associada aos fluxos iônicos rápidos gerados pelo transporte de $\mathrm{Na}^{+}$e $\mathrm{K}^{+}$e $z(\mathrm{t})$ que captura a dinâmica de outros canais lentos. Na composição do potencial de membrana essas variáveis se relacionam de modo não linear, dinâmica que pode ser descrita da seguinte maneira (HINDMARSH; ROSE, 1984):

$$
\left\{\begin{array}{l}
\dot{x}=y-a x^{3}+b x^{2}-z+I ; \\
\dot{y}=c-d x^{2}-y ; \\
\dot{z}=r\left[s\left(x-x_{r}\right)-z\right],
\end{array}\right.
$$

onde $a, b, c, d, s, r, x_{r}$ e $I$ são parâmetros do sistema que, dependendo dos valores adotados, permitem simular um vasto conjunto de comportamentos dinâmicos sendo equivalentes de maneira topológica aos observados no terreno experimental, fazendo do modelo HR um dos mais representativos no estudo qualitativo do fenômeno de bursting neuronal (BUTERA; RINZEL; SMITH, 1999).

O sistema (1) apresenta vários tipos de comportamentos dentre eles, um típico comportamento caótico do potencial de membrana (DE LIMA; CHAVARETTE; ROÉFERO, 2019), isto é, aperiódico e com sensibilidade em relação às condições iniciais adotando-se a estimulação externa constante $I$.

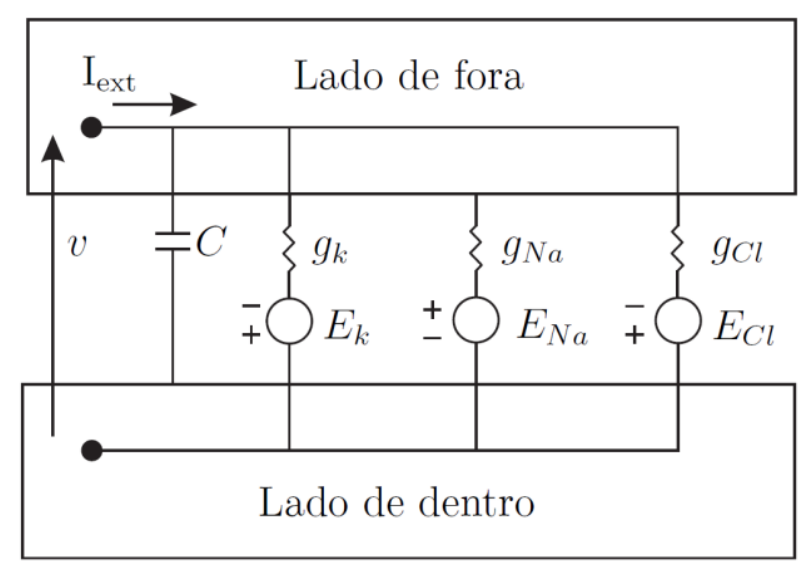

(b)

Fonte: (a) Próprio autor. (b) HODGKIN e HUXLEY, 1952. 


\section{METODOLOGIA}

$\mathrm{Na}$ metodologia de modelagem dos sistemas dinâmicos, as incertezas podem ser consideradas através de uma caracterização probabilística através de intervalos previamente definidos, ou ainda, através da teoria das possibilidades (MOLLER; BEER, 2004).

Para considerar o efeito das incertezas, as mesmas serão associadas à diferença entre os valores reais e os parâmetros do modelo matemático com uma variação de $20 \%$, onde $r(t) \sim u[0,1] \forall t$, tornando-se $\bar{a}=0.8+0.4 r(t)$, $\bar{b}=2.4+1.2 r(t), \bar{c}=0.8+0.4 r(t)$, $\bar{d}=4+2 r(t), \bar{r}=0.0048+0.0024 r(t)$, $\bar{s}=3.2+1.6 r(t), \overline{x_{h}}=-1.248-0.624 r(t) \quad \mathrm{e}$ $\bar{I}=2.4+1.2 r(t)$.

Utilizou-se, nas simulações numéricas, o método de Runge Kutta de 4a ordem. Foi exposto os gráficos do histórico no tempo e plano de fase para os três estados do sistema.

Na realização da simulação numérica do sistema Hindmarsh-Rose utilizou-se a variação temporal de 0 a 1000. Segundo a verificação de controle ótimo (CVETICANIN, 2010), sua função é numericamente calculada por $L(t)=v^{T} Q v$, onde $L(t)$ é definida positiva. Todos os algoritmos foram implementados no software livre Octave.

A análise dos resultados é de natureza qualitativa.

\section{PARÂMETROS INCERTOS}

Sabe-se que os modelos utilizados para projetos são, quase que em sua totalidade, representações aproximadas dos sistemas reais. Geralmente em projetos clássicos são suprimidos o que chamamos de incertezas agregadas nas variações dos parâmetros, variações como, por exemplo, linearização em torno de pontos de operação, alterações de temperatura, desgaste e até mesmo dinâmicas que não foram consideradas na modelagem.

As atuais e inúmeras aplicações na área da engenharia mecânica carecem que os sistemas laborem com parâmetros ótimos sob determinadas condições de operações, como por exemplo a robustez, que comprovam a necessidade do desenvolvimento de modelos numéricos pragmáticos conceituando satisfatoriamente as incertezas nos parâmetros e nas entradas dos sistemas. Pesquisadores têm manifestado, nos últimos anos, grande relevância em otimizações robustas considerando incertezas no desenvolvimento de sistemas mais representativos (HAMI; RADI, 2013).

As incertezas, na área da engenharia mecânica, têm sido consideradas em abundantes ramos como, a dinâmica de estruturas (GHANEM; SPANOS, 2003), dinâmica de rotores (KOROISHI et al., 2012) e robôs manipuladores (LARAMOLINA et al., 2012), entre outros (VICENTE; CHAVARETTE; ROÉFERO, 2019).

\subsection{Projeto de controle de incertezas}

Os parâmetros adimensionais adotados para as simulações numéricas são os $\bar{a}, \bar{b}, \bar{c}$, $\bar{d}, \bar{r}, \bar{s}, \overline{x_{h}}$ e o parâmetro $\bar{I}$ é definido propositalmente para estabelecer o caos no sistema (DE LIMA; CHAVARETTE; ROÉFERO, 2019). Estes parâmetros são definidos de maneira oportuna para uma melhor compreensão do bursting neuronal (HINDMARSH; ROSE, 1984).

Aplicando a teoria proposta por (PEARSON, 1962), podemos projetar o controlador com a função de controle $u \mathrm{e}$ $\dot{x}=A(x)+B(x) u$ onde $A(x) \in R^{n \times n} \quad$ é uma matriz constante, $B(x)$ é a matriz de entrada e o controle ótimo é dado por $u=-R^{-1}(x) B^{T}(x) P(x) x$.

Considerando o sistema equacional (1) juntamente com seu espaço de estado temos: controlabilidade da matriz $R$ do sistema para o par $[A, B]$ é obtida por $R=\left[B|A B| A^{2} B\right]$. Assim, $R=$ [1].

Em seguida, a matriz de co-estado $P(t)$ é dada por

$$
\begin{aligned}
& P=\left[\begin{array}{ccc}
4.8771 & 2.9341 & -8.3505 \\
2.9341 & 2.8859 & -6.8671 \\
-8.3505 & -6.8671 & 19.3971
\end{array}\right] \text { e } \\
& \text { - controle ótimo } \\
& u=-05393 x_{1}-1.0471 x_{2}+4.1795 x_{3} \text {. }
\end{aligned}
$$

\section{RESULTADOS}


Figura 2. Histórico no Tempo

(a)

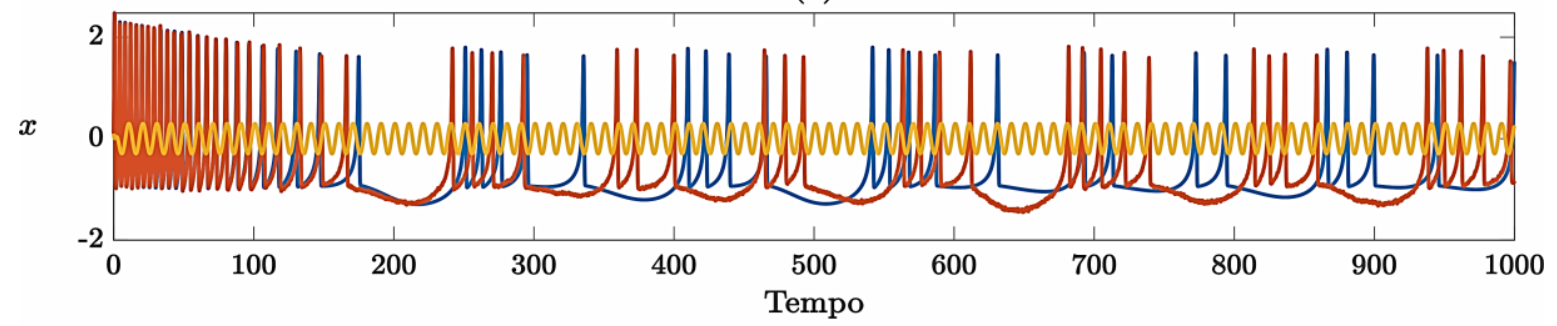

(b)

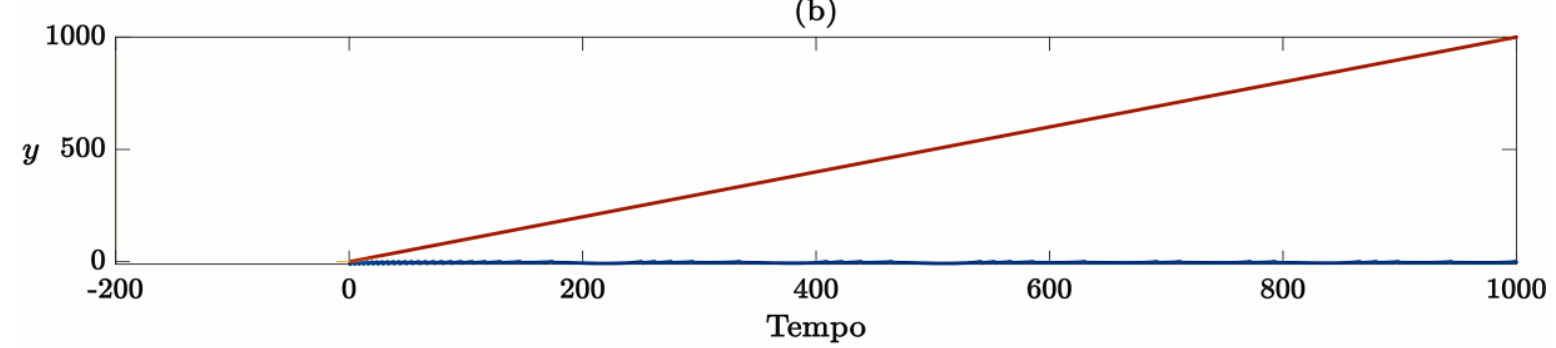

(c)

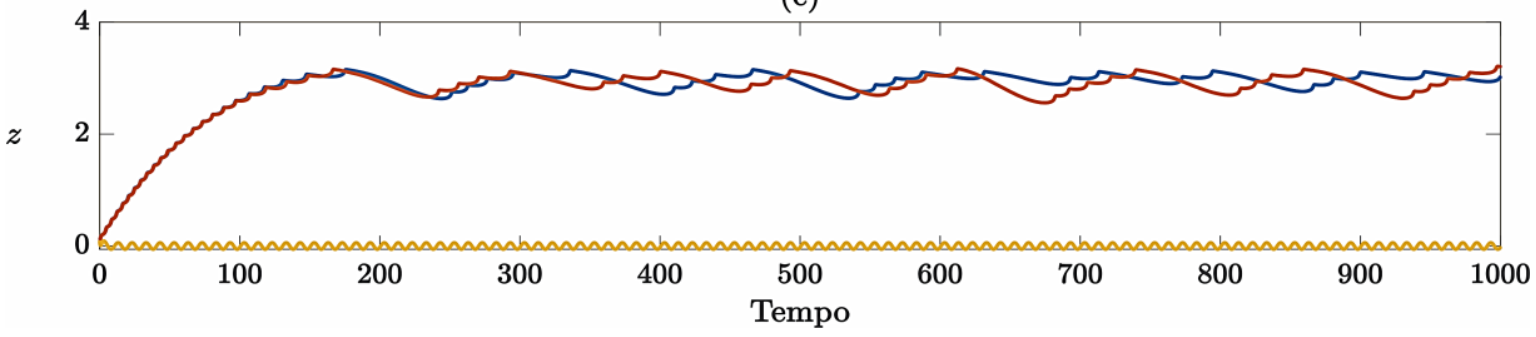

Fonte. Próprio autor

Figura 3. Comportamento dinâmico

(a)

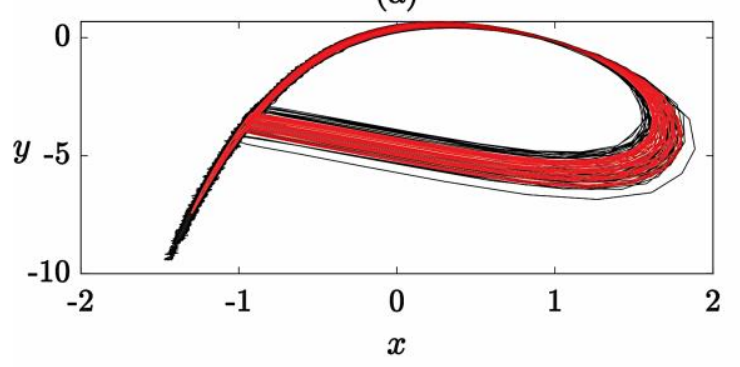

(c)

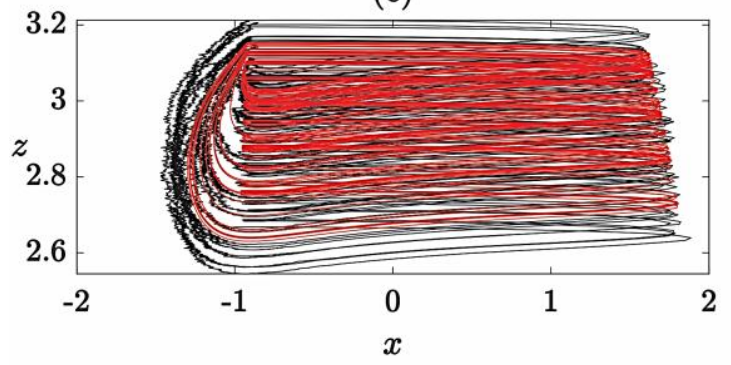

(e)

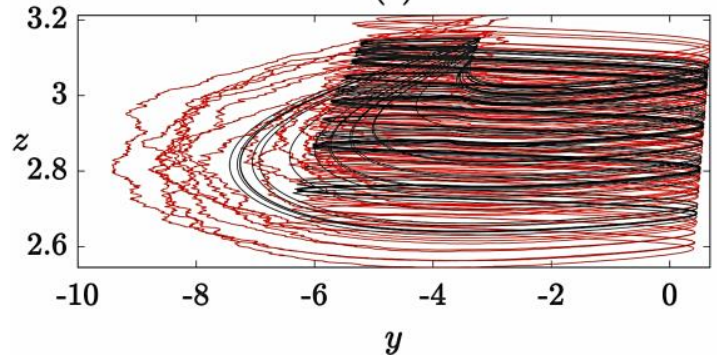

(b)

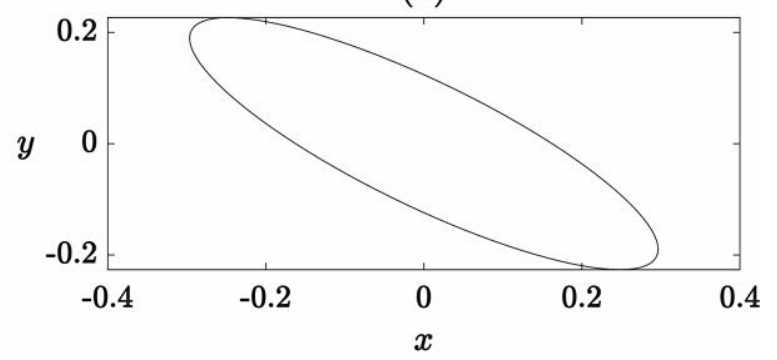

(d)

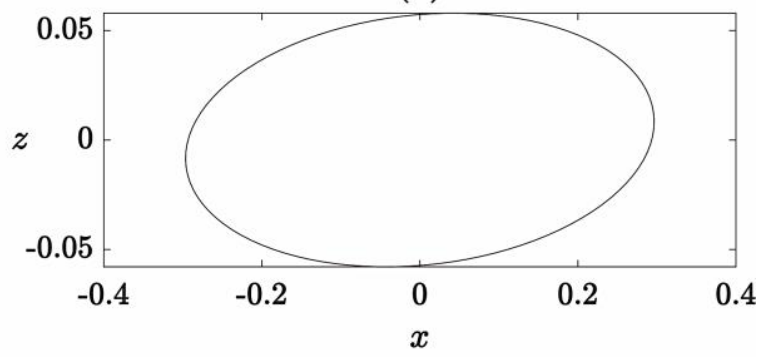

(f)

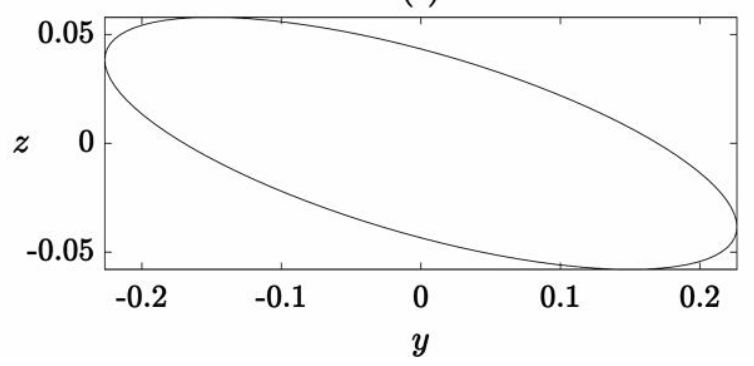

Fonte. Próprio autor 
Na Figura 2 a curva azul representa o sistema não controlado, a curva amarela, por sua vez, está relacionada ao sistema controlado: a) evolução temporal do potencial de membrana $x$; (b) evolução temporal associada aos fluxos iônicos rápidos gerados pelo transporte de $\mathrm{Na}^{+} \mathrm{e}$ $\mathrm{K}^{+}$; e (c) evolução temporal para os canais lentos.

$\mathrm{Na}$ Figura 3 estão representados todos os planos de fase em que: (a) representa o plano $x y$ com o sistema não controlado; (b) representa o plano $x y$ com o sistema controlado; (c) representa o plano $x z$ com o sistema não controlado; (d) representa o plano $x z$ com o sistema controlado; (e) representa o plano $y z$ com o sistema não controlado; e (f) representa o plano $y z$ com o sistema controlado.

\section{CONSIDERAÇÕES FINAIS}

Foi proposto, neste trabalho, um controle do comportamento caótico do sistema HindmarshRose com parâmetros incertos. A técnica de controle linear ótimo utilizando a Equação de Riccati Dependente do Estado (SDRE), foi aplicada ao sistema com o objetivo de solucionar as vibrações incertas no intuito de eliminar o comportamento caótico do potencial de membrana $x$, conduzindo as oscilações a uma vizinhança de uma órbita estável.

Os resultados, ilustrados na Figura 3, demonstram que o controlador proposto obteve sucesso com eficiência e robustez mesmo sendo consideradas incertezas de $20 \%$ sob os parâmetros do sistema.

Os resultados deste trabalho evidenciam a necessidade de aplicar e desenvolver leis de controle cada vez mais robustas com a finalidade de estabilizar todo o processo.

A Organização Mundial da Saúde (OMS) posiciona a epilepsia como uma das doenças neurológias mais comuns do planeta. Estima-se que aproximadamente 50 milhões de pessoas em todo o mundo possuem a doença (LABOISSIÈRE , 2017).

Vale destacar que os resultados expostos neste trabalho podem também ser empregados de maneira análoga em outras áreas correlatas à Engenharia Mecânica como Farmacologia, Engenharia Química, Inteligência artificial e Tecnologia de Circuitos Eletrônicos.

Pesquisas futuras tendem a proporcionar descobertas e métodos mais eficientes no tratamento e combate da epilepsia, como por exemplo, no desenvolvimento de medicamentos que sejam cada vez mais eficientes dispondo de melhor tempo de resposta, implantação de microchips controladores do bursting neuronal dentre outros.

\section{AGRADECIMENTOS}

Agradeço à UFMS.

Agradeço ao Laboratório de Sistemas Complexos (Sisplexos) e ao Programa de PósGraduação em Engenharia Mecânica da Faculdade de Engenharia de Ilha Solteira - UNESP por ceder o espaço físico e o parque computacional para as simulações realizadas neste trabalho.

$O$ segundo autor agradece o apoio financeiro oferecido pela Fundação de Amparo à Pesquisa (FAPESP), através do processo 2019/10515-4 e ao Conselho Nacional de Desenvolvimento Científico e Tecnológico (CNPq), através do processo 312972/2019-9.

\section{REFERÊNCIAS}

BEAR, M. F.; CONNORS, B. W.; PARADISO, M. A. Neurociências: desvendando o sistema nervoso. Porto Alegre: Artmed, 2002.

BUTERA Jr, R. J.; RINZEL, J.; SMITH, J. C. Models of respiratory rhythm generation in the preBotzinger complex. I. Bursting pacemaker neurons. Journal of neurophysiology, v. 82, n. 1 , p. 382-397, 1999.

https://doi.org/10.1152/jn.1999.82.1.382.

CHAVARETTE, F. R. Optimal linear control to parametric uncertainties in a micro electro mechanical system. International Journal of Pure and Applied Mathematics, v. 83, n. 4, p. 539-548, 2013.

https://doi.org/10.12732/ijpam.v83i4.2.

CLOUTIER, J. R.; D'SOUZA, C. N.; MRACEK, C. P. Nonlinear regulation and nonlinear $\mathrm{H} \infty$ control via the state-dependent Riccati equation technique: Part 1, theory. In: INTERNATIONAL CONFERENCE ON NONLINEAR PROBLEMS IN AVIATION AND AEROSPACE. 1996. Daytona Beach, FL. Proceedings [...]. Daytona Beach, FL: Embry Riddle University, 1996. p. 117-131.

CVETICANIN, L. Dynamics of the non-ideal mechanical systems: A review. Journal of the 
Serbian Society for Computational Mechanics, $v$. 4, n. 2, p. 75-86, 2010.

DE LIMA, R. S.; CHAVARETTE, F. R.; ROÉFERO, L. G. P. Estudo do Comportamento Dinâmico do Modelo Neuronal de Hindmarsh-Rose. Colloquium Exactarum, v. 11, n. 4, p. 122-130, 2019.

https://doi.org/10.5747/ce.2019.v11.n4.e301

GHANEM, R. G.; SPANOS, POL D. Stochastic finite elements: a spectral approach. Courier Corporation, 2003.

HAMI, A. E.; RADI, B. Uncertainty and Optimization in Structural Mechanics. Nova Jersey: John Wiley \& Sons, 2013.

HINDMARSH, J. L.; ROSE, R. M. A model of neuronal bursting using three coupled first order differential equations. Proceedings of the Royal society of London. Series B. Biological sciences, v. 221 , n. 1222 , p. 87-102, 1984.

https://doi.org/10.1098/rspb.1984.0024.

HODGKIN, A. L.; HUXLEY, A. F. A quantitative description of membrane current and its application to conduction and excitation in nerve. The Journal of physiology, v. 117, n. 4, p. 500544 , 1952. https://doi.org/10.1113/iphysiol.1952.sp004764.

JUN, M. et al. Control Chaos in Hindmarsh-Rose Neuron by Using Intermittent Feedback with One Variable. Chinese Physics Letters, v. 25, n. 10, p. 3582, 2008. https://doi.org/10.1088/0256$\underline{307 X / 25 / 10 / 017}$

KOROISHI, E.H.; CAVALINI Jr, A.A.; DE LIMA, A.M.G.; Steffen Jr, V. Stochastics modeling of exible rotors. Journal of the Brazilian Society of Mechanical Sciences and Engineering, 34(Special Issue):597-603, 2012.

http://dx.doi.org/10.1590/S1678-

$\underline{58782012000600006}$

LABOISSIÈRE, P. Cerca de 50 milhões de pessoas em todo o mundo têm epilepsia, alerta OMS. Agência Brasil, $2017 . \quad$ Disponível em: $<$ https://agenciabrasil.ebc.com.br/geral/notic ia/2017-02/cerca-de-50-milhoes-de-pessoas-emtodo-o-mundo-tem-epilepsia-alerta-oms >. Acesso em 13/02/2021.
LARA-MOLINA, F. A.; ROSARIO, J. M., DUMUR, D., WENGER, P. Generalized predictive control of parallel robots. In: KOZLOWSKI, K. (ed.). Robot Motion and Control 2011. London: Springer, 2012. Cap. 13, p. 159-169. https://doi.org/10.1007/978-1-4471-2343-9 13

LORENZ, E. N. Section of planetary sciences: The predictability of hydrodynamic flow* $\dagger$. Transactions of the New York Academy of Sciences, v. 25, n. 4, Series II, p. 409-432, 1963. https://doi.org/10.1111/i.21640947.1963.tb01464.x.

MOLLER, B.; BEER, M. Fuzzy Randomness, Uncertainty in Civil Engineering and Computational Mechanics. Springer-Verlag, 2004. https://doi.org/10.1007/978-3-662-073582

MONTEIRO, L. H. A. Sistemas dinâmicos. São Paulo: Livraria da Física, 2002.

MRACEK, C. P.; CLONTIER, J. R.; D'SOUZA, C., A. A new technique for nonlinear estimation. In: IEEE INTERNATIONAL CONFERENCE ON CONTROL APPLICATIONS IEEE INTERNATIONAL CONFERENCE ON CONTROL APPLICATIONS HELD TOGETHER WITH IEEE INTERNATIONAL SYMPOSIUM ON INTELLIGENT CONTRO. 1996, Dearborn, Michigan. Proceeding [...]. Dearborn, Michigan: IEEE, 1996. p. 338-343.

https://doi.org/10.1109/CCA.1996.558760.

PALUS, M. et al. Synchronization and information flow in EEGs of epileptic patients. IEEE Engineering in Medicine and Biology Magazine, v. 20, n. 5, p. 65-71, 2001. https://doi.org/10.1109/51.956821.

PEARSON, J. D. Approximation methods in optimal control I. Sub-optimal control. International Journal of Electronics, v. 13, n. 5, p. 453-469, 1962.

https://doi.org/10.1080/00207216208937454.

SHAWKY, A. M. et al. Position control of flexible manipulator using non-linear $\mathrm{H} \infty$ with statedependent Riccati equation. Proceedings of the Institution of Mechanical Engineers, Part I: Journal of Systems and Control Engineering, v. 221, n. 3, p. 475-486, 2007.

https://doi.org/10.1243/09596518JSCE313. 
VICENTE, J. P. G.; CHAVARETTE, F. R.; ROÉFERO, L. G. P. Chaos Control via Mathieu-Van der Pol System and Linear Optimal Control Design with a Non-ideal Excitation and Parametric Uncertainties. Revista Internacional de Metodos Numericos para Calculo y Diseno en Ingenieria, v. 35, p. 37-47, 2019.

http://dx.doi.org/10.23967/j.rimni.2019.08.001.

WERNLI, A.; COOK, G. Suboptimal control for the nonlinear quadratic regulator problem. Automatica, v. 11, n. 1, p. 75-84, 1975. https://doi.org/10.1016/0005-1098(75)90010-2. 